\title{
CENTRAL RETINAL VEIN OCCLUSION
}

\author{
Bharati Gogoi1, Farhana Rahman²
}

1 Professor, Department of Ophthalmology, RIO, GMCH, Guwahati, Assam.

2Postgraduate Student, Department of Ophthalmology, RIO, GMCH, Guwahati, Assam.

\section{BACKGROUND}

\section{ABSTRACT}

CRVO is a retinal vascular occlusive disorder seen commonly in older adults and is often associated with systemic vascular disease. The patient usually presents with unilateral painless loss of central vision. It is characterised by haemodynamic changes leading to thrombus formation in the central retinal vein. Fundoscopic findings including optic disc and retinal oedema, dilated tortuous retinal veins with fibrous sheathing, extensive superficial and deep retinal haemorrhages, cotton wool spots and appearance of collaterals at the optic disc are its prominent diagnostic signs. FFA shows a marked delay in filling up of retinal veins with areas of blocked fluorescence corresponding to areas of extensive haemorrhages. Although, most eyes recover with a good vision, about one-fifth have significant visual loss and many suffer ocular sequelae. Many treatment modalities such as intravitreal injections, laser photocoagulation, invasive procedures like radial optic neurotomy and vitrectomy have been tried for this entity, but no conclusive evidence exists that any treatment alters its natural history.

\section{KEYWORDS}

Ischaemia, Macular Oedema, Haemorrhage, Neovascularisation.

HOW TO CITE THIS ARTICLE: Gogoi B, Rahman F. Central retinal vein occlusion. J. Evolution Med. Dent. Sci. 2018;7(18):2288-2290, DOI: $10.14260 /$ jemds/2018/514

\section{BACKGROUND}

Central retinal vein occlusion (CRVO) is the third most common vascular retinal disorder after diabetic retinopathy and branch retinal vein occlusion. It is caused either from thrombus formation within the central retinal vein or vascular compression. 1,2

\section{Pathogenesis}

Central retinal vein occlusion is the occlusion of central vein at or just behind the level of lamina cribrosa. There are certain anatomical factors that predispose the central retinal vein occlusion. The central retinal artery and central retinal vein are bounded by a common adventitial sheath. The flow of blood through the central retinal vein becomes increasingly turbulent, as the vein progressively narrows at the lamina cribrosa. This turbulence damages the endothelium in the retrolaminar vein, which exposes collagen and initiates platelet aggregation and thrombosis. At the level of lamina cribrosa, the central retinal vein and artery share a common adventitial sheath. It occurs that atherosclerotic changes in the central retinal artery transforms it into a rigid structure that presses upon the pliant central retinal vein. This results in haemodynamic alterations, increased turbulence of the blood flow, resultant endothelial damage and thrombus formation. The central retinal vein has no space to move or expand because of the restricting adventitial sheath..$^{3,4}$

\section{Classification}

Hayreh advocated that the disease CRVO has actually two distinct entities. Haemorrhagic retinopathy or Ischaemic

'Financial or Other Competing Interest': None.

Submission 07-03-2018, Peer Review 11-04-2018,

Acceptance 18-04-2018, Published 30-04-2018.

Corresponding Author:

Dr. Farhana Rahman

Department of Ophthalmology,

RIO, GMCH, Guwahati, Assam, India.

E-mail: drfarhanarahman9@gmail.com

DOI: $10.14260 /$ jemds $/ 2018 / 514$

\section{(c) $(\mathbf{F})$}

CRVO is the term for the more severe form of CRVO with high risk for neovascular complications. Venous stasis retinopathy or non-ischaemic CRVO is the term used to describe the milder form of the disease, which have a relatively benign course. ${ }^{5}$

\section{CLINICAL PRESENTATION}

In occlusion of the central retinal vein, the patient typically present with sudden loss of central vision in the affected eye. This however at times may be gradual, although the visual acuity may be reduced to the perception of hand movement or light perception. Some patients present with pain and evidence of iris neovascularisation and neovascular glaucoma at the time of their initial examination. This usually follows a loss of vision, roughly 3 to 4 months before the onset of pain. These eyes typically have corneal oedema, high intraocular pressure and extensive iris neovascularisation. ${ }^{6}$ Classically, NVG occurs within 3 months of the CRVO, hence the name 100 day glaucoma. ${ }^{7}$

Ophthalmoscopically, it reveals the classic appearance of oedema of the optic disc and retina, marked dilatation and tortuosity of the retinal veins, extensive superficial and deep retinal haemorrhages radiating outward from the optic disc and extending into the periphery in all quadrants. ${ }^{8}$

Based on the clinical course, CRVO is highly variable. In some cases, the signs of occlusion disappear in a few months leaving little signs. Intermediate cases may leave persistent haemorrhage, macular oedema and microvascular abnormalities such as microaneurysms. In ischaemic disease, complications may develop such as rubeosis iridis, neovascular glaucoma, retinal or disc neovascularisation and vitreous haemorrhage.

\section{Associated Conditions}

CRVO is associated with both ocular and systemic conditions. A high prevalence of systemic hypertension (about 60\% of cases in several large series) has consistently been associated with CRVO. Other conditions associated are diabetes, cardiovascular disease and peripheral vascular disease. ${ }^{9}$ 


\section{Methods of Evaluation 10 \\ Visual Acuity}

In acute CRVO, visual acuity ranges from normal to just the ability to see hand movements.

\section{Afferent Pupillary Defect}

A quantitative measurement of relative afferent pupillary defect in eyes with CRVO has been shown to correlate well with retinal ischaemia and to be a good predictor of eyes at risk for neovascular complications. $90 \%$ of eyes designated nonischaemic by other criteria had relative afferent defects of 0.3 log units or less. $91 \%$ of eyes with ischaemic CRVO had relative afferent defects of $1.2 \mathrm{log}$ units or more.

\section{IOP}

Glaucoma and elevated IOP are risk factors for CRVO. Immediately after CRVO, the IOP is typically slightly lower in the affected eye than in the fellow eye. This relative pressure difference diminishes with time and symmetry returns over the ensuing weeks to months.

\section{Visual Field}

Central and peripheral visual field abnormalities are more common and more severe in ischaemic than in non-ischaemic CRVO.

\section{Fundus Findings}

Cotton-wool spots are an indicator of ischaemia. The presence of 10 or more cotton-wool spots was found to be a risk factor for the development of rubeosis iridis.

The resolution of fundus finding is quite variable and unpredictable. The retinal haemorrhages tend to decrease in number over many months and may disappear entirely; however, some may persist for years, particularly in periphery. The dilatation and tortuosity of the involved retinal veins typically diminish over time and marked fibrous sheathing of the retinal veins and arteries may develop. Disc oedema regresses, in ischaemic cases disc pallor may develop. Collateral vessels at the optic disc may develop in mild or severe occlusion.

\section{Fundus Fluorescein Angiography}

A characteristic finding in CRVO are the result of changes in vascular calibre, abnormal vascular permeability and closure of the retinal capillaries. FFA shows a marked delay in filling up of retinal veins with areas of blocked fluorescence corresponding to areas of extensive haemorrhages.

Scanning laser ophthalmoscopic fluorescein angiograph and digital image analysis have demonstrated reduced perifoveal capillary blood velocity and enlargement of the foveal avascular zone.

\section{Electroretinography}

Indicators of ischaemia are reduced B-wave amplitude, reduced $b$ : a wave ratio and prolonged $b$-wave implicit time. Various other ERG parameters have been evaluated and found to have variable reliability in diagnosing retinal ischaemia and predicting ocular neovascularisation. These include b-wave implicit time, b/a ratio of scotopic and photopic ERG, $30 \mathrm{~Hz}$ flicker latency etc. ${ }^{11}$

\section{Complications ${ }^{10}$}

1. Macular Oedema: Macular oedema may occur and is one of the leading causes of visual loss in central retinal vein occlusion. It results from leakage of the perifoveal vessels secondary to hydrostatic stress and ischaemia. The oedema typically takes on clinical and fluorescein angiographic appearance of CME and may be transient or persistent.

2. Haemorrhage: In acute CRVO, the amount of haemorrhage is highly variable. If haemorrhage occurs in the fovea, visual acuity will be affected. Occasionally, acute CRVO may lead to vitreous haemorrhage, which if severe enough may obscure the fundus and diagnosis.

3. Ischaemia: Ischaemia refers to closure of the retinal capillaries and is angiographically described as capillary dropout or capillary non-perfusion.

4. Neovascularisation: The proliferation of new blood vessels is a complication of ischaemic processes affecting the retina. Retinal ischaemia leads to neovascularisation, which may take place on the optic disc, on the retina, on the angle and on the iris (Rubeosis iridis).

5. Retinal Detachment: Rhegmatogenous retinal detachment may occur. Other vascular complication such as microaneurysm typically develops in the retina after venous occlusion.

Collateral channels between the retinal and the ciliary circulations (sometimes called optociliary vessels) develop in about $50 \%$ of cases. They appear as tortuous vascular loops on or adjacent to the optic disc. They may be confused with disc neovascularisation, but can easily be distinguished by their larger calibre and lack of leakage on fluorescein angiography.

\section{Management}

1. Control of associated systemic conditions like hypertension and diabetes.

2. Intravitreal Triamcinolone Acetonide (IVTA)

Injection of triamcinolone $(0.1 \mathrm{~mL} / 4 \mathrm{mg})$ into vitreous cavity through pars plana is effective to treat macular oedema in both ischaemic and non-ischaemic CRVO. The role of corticosteroids is based primarily on the inhibition of biosynthetic pathways of leukotrienes and prostaglandins, the inflammatory mediators implicated in pathogenesis of macular oedema. The main drawback of an injection of triamcinolone is the post treatment recurrence of macular oedema, requiring repeat triamcinolone injection, every 3 - 6 months.

3. Anti-VEGF Agents ${ }^{12,13}$

VEGF is involved in the pathogenesis of macular oedema. It also produces pathological neovascularisation. AntiVEGF inhibits abnormal vessel growth, while sparing normal vessels.

\section{Panretinal Photocoagulation (PRP)}

It is recommended if iris/ angle neovascularisation is detected (the central vein occlusion study used two clock hours of NVI).

5. Laser Induced Anastomosis ${ }^{4}$ between a Retinal Vein and the Choroid

It is a procedure to bypass the central retinal vein outflow. At a site at least three disk diameters away from the optic nerve and usually along the inferotemporal vein, a green argon laser using a power of 2.5 to $3.5 \mathrm{~W}$ creates a small 
spot to rupture Bruch's membrane and a second spot to rupture an adjacent venule.

6. Thrombolytic Agents such as t-PA (Tissue Plasminogen Activator)

It is used via the systemic, intravenous ${ }^{14}$ and retinal venous route. 15 Injection of t-PA into a peripapillary branch retinal vein targets the thrombus at the lamina cribrosa.

7. Vitrectomy with peeling of the posterior hyaloid face. It may decrease macular oedema.

8. Radial Optic Neurotomy ${ }^{16,17}$

It is a procedure to decompress the central retinal vein at the lamina cribrosa to release the 'napkin-ring/bottleneck' constriction. A radial incision of the lamina and sclera is made at the nasal edge of the optic disc. The mechanisms for its functional results include relief of mechanical pressure exerted on the central retinal vein by the distended optic nerve, the creation of chorioretinal anastomosis and the enhancement in the exchange between retina and vitreous cavity secondary to posterior hyaloid peeling, thereby reducing oedema.

\section{REFERENCES}

[1] Klien BA, Olwin JH. A survey of the pathogenesis of retinal venous occlusion, emphasis upon choice of therapy and an analysis of the therapeutic results in fifty-three patients. AMA Arch Ophthalmol 1956;56(2):207-47.

[2] Klien BA. Sidelights on retinal veinous occlusion. Am J Ophthalmol 1966;61(1):25-36.

[3] The Central Vein Occlusion Study Group M report. Evaluation of grid pattern photocoagulation for macular edema in central vein occlusion. Ophthalmology 1995;102(10):1425-33.

[4] McAllister IL, Constable IJ. Laser-induced chorioretinal venous anastomosis for treatment of non-ischemic central retinal vein occlusion. Arch Ophthalmol 1995;113(4):456-62.

[5] Hayreh SS. Classification of central retinal vein occlusion. Ophthalmology 1983;90(5):458-74.

[6] Weiss DI, Shaffer RN, Nehrenberg TR. Neovascular glaucoma complicating carotid-cavernous sinus fistula. Arch Ophthalmol 1963;69:304-7.
[7] Hayreh SS, Rojas P, Podhajsky P, et al. Ocular neovascularization with retinal vascular occlusion-III. Incidence of ocular neovascularization with retinal vein occlusion. Ophthalmology 1983;90(5):488-506.

[8] Hayreh SS. Optic disc vasculitis. Br J Ophthalmol 1972;56(9):652-70.

[9] Hayreh SS, Podhajsky PA, Zimmeman MB. Retinal artery occlusion: associated systemic and ophthalmic abnormalities. Ophthalmology 2009;116(10):1928-36.

[10] Albert \& Jakobiec's Principles \& practice of ophthalmology. Venous Occlusive diseases of the retina. $3^{\text {rd }}$ edn. Saunders Elsevier 2008: p. 1887.

[11] Subhadra Jalali S, TP Das. Modern ophthalmology. Chapter - 192. Retinal venous occlusive diseases. Vol. 3. 3rd edn. 2005: p. 1584.

[12] Boyd SR, Zachary I, Martin JF, et al. Aqueous vascular endothelial growth factor correlates with clinical course of disease in human ischaemic central retinal vein occlusion. Invest Ophthalmol Vis Sci 1999;40:S376.

[13] Boyd SR, Zachary I, Chakravarthy U, et al. Correlation of increased vascular endothelial growth with neovascularization and permeability in ischemic central vein occlusion. Arch Ophthalmol 2002;120(12):1644-50.

[14] Weizer JS, Fekrat S. Intravitreal tissue plasminogen activator for the treatment of central retinal vein occlusion. Ophthalmic Surg Lasers Imaging 2003;34(4):350-2.

[15] Weiss JN, Bynoe LA. Injection of tissue plasminogen activator into a branch retinal vein in eyes with central retinal vein occlusion. Ophthalmology 2001;108(12):2249-57.

[16] Garcia-Arumii J, Boixadera A, Martinez-Castillo V, et al. Chorioretinal anastomosis after radical optic neurotomy for central retinal vein occlusion. Arch Ophthalmol 2003;121(10):1385-91.

[17] Opremak EM, Bruce RA, Lomeo MD, et al. Radical optic neurotomy for central retinal vein occlusion: a retrospective pilot study of 11 consecutive cases. Retina 2001;21(5):408-15. 